\title{
Three-dimensional scattering matrix simulation of resonant tunnelling via quasi-bound states in vertical quantum dots
}

\author{
H. Mizuta* \\ Hitachi Cambridge Laboratory, Hitachi Europe Ltd., Cambridge, UK
}

Received 1 March 1999; accepted 8 March 1999

\begin{abstract}
This paper presents a numerical simulation technique for quantum-dot-based electronic devices based on the three-dimensional (3D) scattering matrix (S-matrix) theory. Starting from the 3D time-independent Schrödinger equation with scattering boundary conditions, the multi-mode S-matrix and transmission rates are derived and the tunnelling current is calculated based on the global coherent tunnelling model. The present simulation technique is applied for zero-dimensional (0D) resonant tunnelling diodes (RTDs). The effects of a complex mixture of lateral mode conserving and non-conserving tunnelling processes on the $I-V$ characteristics are investigated in terms of multimode transmission rates and quasi-bound electronic states at resonance. The simulation is also used for analysing resonant tunnelling (RT) assisted by ionised impurities in a quantum dot. By introducing ionised impurities in the quantum dot region, a new type of RT via singleimpurity-induced quasi-bound states is investigated. (C) 1999 Elsevier Science Ltd. All rights reserved.
\end{abstract}

Keywords: Zero-dimensional resonant tunnelling structures; Scattering matrix; Electron confinement; Lateral mode mixing; Random ionised impurity

\section{Introduction}

Recent rapid advances in atomic-scale crystal growth and nanofabrication techniques have enabled us to realise various kinds of three-dimensional (3D) electron confinement in semiconductors. For electronic device applications these confinement structures are often formed in a conduction channel of the device, and new phenomena caused by the confined electrons are utilised to create novel device functions. Typical structures are vertical zero-dimensional (0D) resonant tunnelling (RT) structures [1-9] and lateral two-dimensional (2D) electron gas (2DEG) structures with patterned gate electrodes. These structures are regarded as a virtually isolated quantum dot, which is weakly coupled to reservoirs and so are well suited to investigate transport properties of a small number of electrons. In these structures effects of the 3D energy quantisation and Coulomb blockade on electron transport become significant, and it has become of great interest to investigate new memory and logic applications by using these unique phenomena.

Numerical simulation of carrier transport in the quasi-0D structures has also become increasingly important especially for designing and analysing new functional elec-

\footnotetext{
* Tel.: + 44-1223-442903; fax: + 44-1223-467942.
}

E-mail address: mizuta@phy.cam.ac.uk (H. Mizuta) tronic devices. A proper description of quantum transport in nanostructures is given in terms of a non-equilibrium quantum transport theory, and various approaches have been reported such as non-equilibrium Green's function theory [10], density matrix theory [11], and Wigner distribution function theory [12]. These approaches have been studied intensively and applied for some simple structures such as a single heterojunction and a double-barrier (DB) RTD. However, such sophisticated approaches have not been used for simulating more complicated structures because of difficulty in implementation. Instead, several numerical modelling methods have been developed for the quasi-0D structures by introducing various simplifications. First, a 3D scattering matrix (S-matrix) theory has recently been adopted to analyse the 3D quantisation effects on tunnelling currents in 0D RTDs. This approach facilitates a detailed analysis of multi-mode RT processes as well as 0D quasibound states in the quantum dots. As the tunnelling current is calculated assuming global coherent tunnelling of electrons, inelastic scattering processes and many-body effects cannot be studied in this approach. Nevertheless, this method has been used as a powerful tool for analysing the effects of lateral mode mixing $[13,14]$ and ionised-impurityassisted tunnelling [15] on the $I-V$ characteristics for the $0 \mathrm{D}$ RTDs. Second, the N-electron model Hamiltonian has been recently solved for a quasi-0D system with a simple 


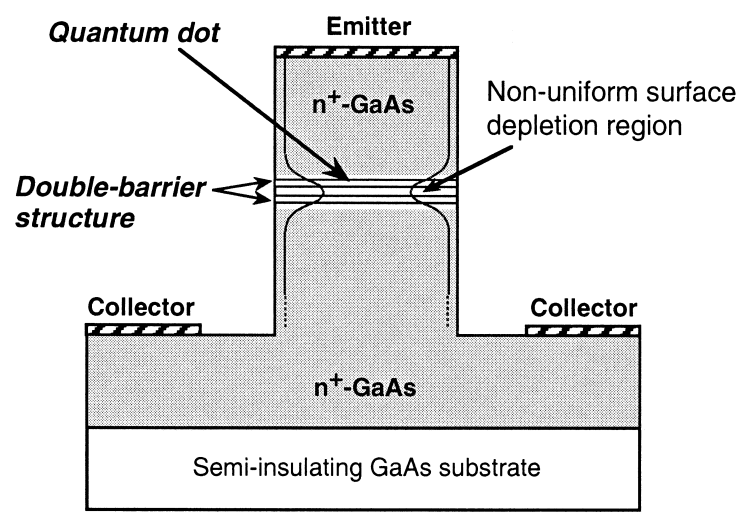

(a) 2-terminal pillar RTD

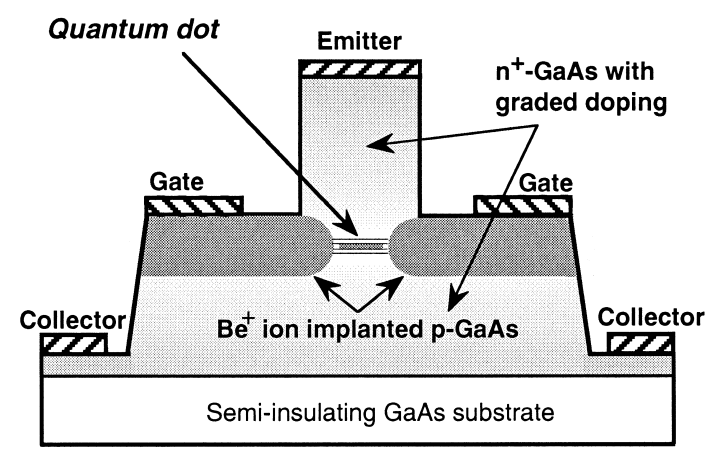

(b) 3-terminal RTD with an implanted gate

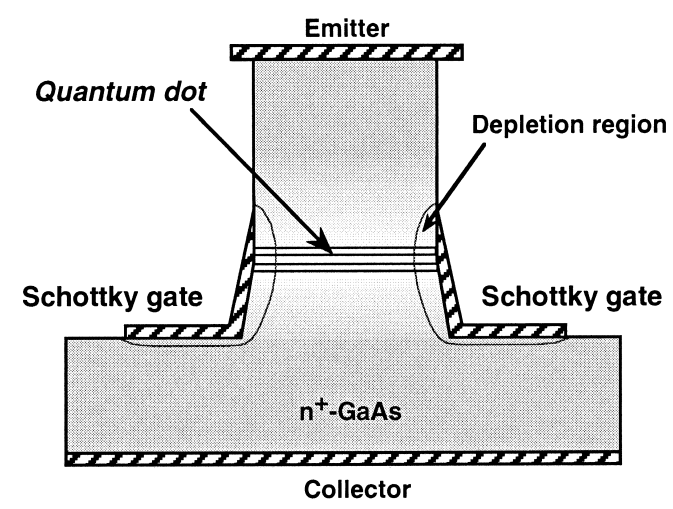

(c) 3-terminal RTD with a Schottky gate

Fig. 1. Schematic cross-sectional views of three typical 0D RTD structures: (a) two-terminal etched RTD; (b) three-terminal RTD with an implanted inplane gate; and (c) three-terminal RTD with a Schottky side gate.

harmonic confinement potential $[16,17]$. Combined with the tunnelling Hamiltonian model, this approach enables calculating many-body electronic states beyond a mean field approximation and the details of the Coulomb blockade oscillation. Finally, it should also be noted that, in addition to these microscopic numerical approaches, a macroscopic

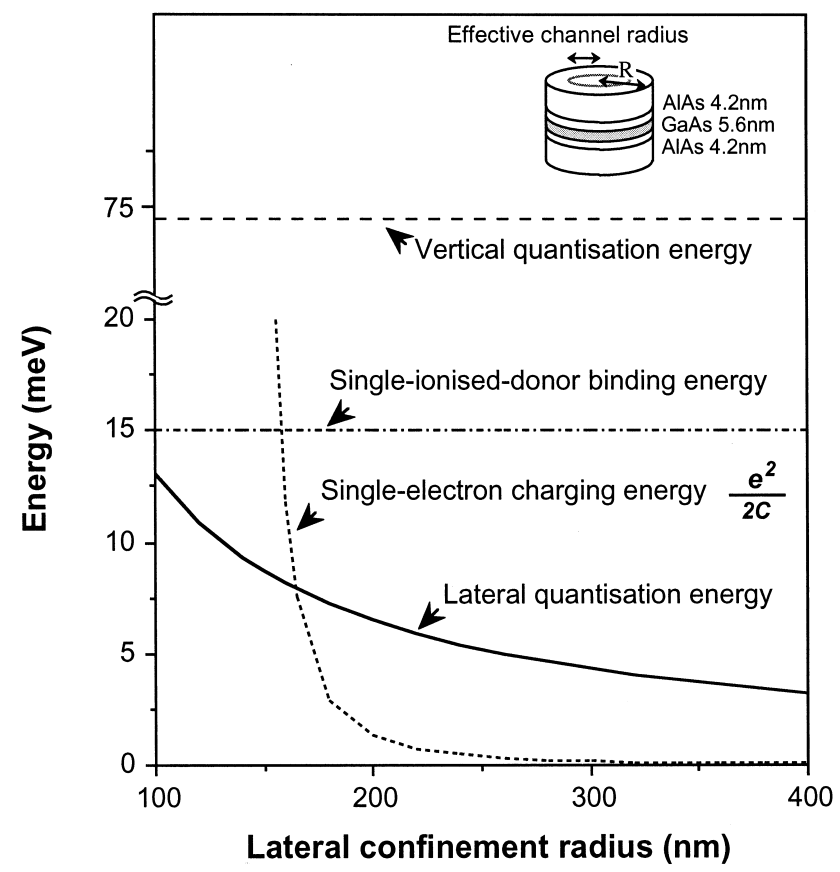

Fig. 2. Various quatisation energies estimated for and AlGaAs/GaAs/ AlGaAs quantum dot as a function of lateral confinement radius.

equivalent circuit simulation has become very popular as a handy design tool for the quantum dot devices and circuits. In this simulation all the quantum mechanical natures of electrons in the quantum dot are modelled by using two macroscopic parameters, tunnel resistance and capacitance.

This paper is intended to present a review of the recent works based on the 3D S-matrix theory mentioned above. Throughout the paper the GaAs-based OD RTDs are employed as an object of numerical analysis as there have been an enormous amount of experimental studies for this device. In Section 2 we introduce various 0D RTD structures and give a brief review of the key transport physics in this device. Section 3 is devoted to describe the 3D S-matrix theory and to derive expressions of multi-mode transmission rates. Then the multi-mode RT and associated quasi-bound states in a quantum dot are numerically analysed in Section 4. Finally more complex RT induced by ionised background impurities in a quantum dot is investigated in Section 5.

\section{Brief review of experimental studies of OD RTDs}

The 0D RTDs consist of double- (or multiple-) barrier RT structures with various types of lateral confinement. Fig. 1(a)-(c) show three typical structures. The simplest structure is a pillar-shaped two-terminal device [1] with a diameter of a few tens nanometers. In this structure the lateral confinement is formed by a fixed surface depletion region as shown in Fig. 1(a). As the surface depletion length varies along the channel depending on local doping concentration, and so an hour-glass shaped non-uniform 
confinement is formed. The 0D RTDs in Fig. 1(b) and (c) have gate electrodes, and lateral size of the quantum dot is variable via gate bias. In the structure shown in Fig. 1(b) $[18,19]$, p-type $\mathrm{Be}^{+}$ion implanted gate regions are employed, which form the lateral PN junction. The implanted regions are prepared so that the peak of dopant distribution is aligned to the DB structure. Because of diffusion of implanted ions, lateral size of the quantum dot becomes much smaller than the emitter pad size, and the lateral quantisation effects can be observed even for devices with a fairly large emitter size [19]. The structure shown in Fig. 1(c) has a Schottky side gate formed by using a shadow evaporation technique with the top contact being used as the mask $[8,9]$. The undercut of the mesa structure has been achieved by combining a chemically assisted ion beam etching and an isotropic wet etch. The device with a diameter down to $0.5 \mu \mathrm{m}$ has been reported for this structure.

In the 0D RTD structures an interplay of lateral quantisation and Coulomb blockade can be observed depending on the structural parameters. Fig. 2 show various quantisation energies as a function of a lateral confinement radius, $R$, estimated for a $\operatorname{AlAs}(10 \mathrm{~nm}) /$ $\operatorname{GaAs}(5.6 \mathrm{~nm}) / \operatorname{AlAs}(10 \mathrm{~nm})$ quantum dot by using a simple parabolic confinement model. The largest quantisation energy is a vertical quantisation energy (a broken line) which is determined by a DB structure and results in the negative differential conductance (NDC) observable at room temperature. The lateral quantisation energy (a solid line) and single-electron charging energy (a dotted line) are roughly proportional to $1 / R$ and $1 / R^{2}$, respectively. These two energies, therefore, exhibit a crossover when $R$ is decreased. In the $0 \mathrm{D}$ RTDs the quantum dots are usually formed by using undoped semiconductors, but there may exist background impurities, either as residual dopants in a growth process and/or as the ones segregated from the contact regions. In these circumstances a binding energy due to an ionised single impurity (a dotted broken line) should also be taken into account, which can be of order of $10 \mathrm{meV}$ [20].

The lateral quantisation effects were first studied by using the pillar-type AlGaAs/InGaAs/AlGaAs structure [1] (Fig. 1(a)). A clear fine structure was observed in the $I-V$ characteristics even at rather high temperatures $(\sim 77 \mathrm{~K})$ superimposed on the usual NDC of 2D-RTDs. The experimental data was explained by assuming sequential electron tunnelling from quantised lateral levels in the emitter region to quantum dot levels in the well, and the observed fine peaks were attributed to tunnelling in which lateral momentum is not conserved $[2,21]$. The difficulty in analysing the data stems from the non-uniform lateral confinement potential (see Fig. 1(a)). Non-uniform confinement in the quantum dot causes wavefunction mismatch and lateral-mode mixing. Further study of the lateral quantisation effects has been done by using the AlAs/GaAs/AlAs 0D RTD structure $[18,19]$ shown in Fig. 1(b). As a result of the graded doping used in the emitter and collector contact layers, a virtually flat lateral confinement potential is achieved around the quantum dot, which can prevent complex lateral-mode mixing. Multiple current peaks have been observed in the current pinch-off regime, and the gate bias dependence of these peak voltages has been found to be consistent with a simple lateral confinement model.

A series of plateau-like structures have also been observed before a main current peak for the same 0D RTD structures (Fig. 1(b)) with a larger emitter size. The current plateaux have been found basically independent of the gate bias, i.e. the lateral confinement size, unlike those resulting from the lateral confinement or Coulomb blockade. From the background donor concentration, the number of the donor sites was estimated to be from 1 to 5 for these devices, and the observed fine structures were attributed to a few ionised donors in the quantum dot $[13,15]$. Those ionised donors will result in a localised potential well and associated bound states, which give rise to RT at lower bias voltages than the threshold voltage.

As shown in Fig. 2, Coulomb blockade phenomenon may be observed for smaller devices at lower temperatures. For studying Coulomb blockade, the 0D RTDs with an asymmetric DB structure have been preferably used as charge build-up is enhanced in those structures when an opaque barrier is placed on the collector side. The pillar-type 0D RTDs (Fig. 1(a)) with asymmetric AlGaAs/GaAs/AlGaAs DB structures have been used in the early studies $[22,23]$. In such 0D RTDs the effect of lateral quantisation and that of the Coulomb blockade were observed separately in two bias directions: current steps attributable to Coulomb blockade were observed when the transparent AlGaAs barrier is located on the emitter side while multiple current peaks due to the lateral quantisation observed in a reverse direction. Recent experiments performed by using the OD RTD structure shown in Fig. 2(c) have clearly showed the Coulomb blockade oscillation in the conductance as a function of gate bias. By analysing the details of the oscillation, it has been found that the energy levels in the quantum dot are filled with electrons according to Hund's rule, like artificial atoms [9].

Among these various phenomena in the OD RTDs, this paper focuses on the effects of the lateral confinement and lateral-mode mixing (Section 4) and those of the ionised-impurity-induced RT (Section 5) on the $I-V$ characteristics.

\section{Numerical simulation based on 3D S-matrix theory}

The 3D S-matrix theory is an expansion of the transfer matrix method for one-dimensional (1D) tunnelling problems to more general 3D systems. We start from the 
3D time-independent Schrödinger equation

$$
\begin{aligned}
- & \frac{\hbar^{2}}{2 m^{*}}\left(\frac{\partial^{2}}{\partial x^{2}}+\frac{\partial^{2}}{\partial y^{2}}+\frac{\partial^{2}}{\partial z^{2}}\right) \Psi(x, y, z)+V(x, y, z) \Psi(x, y, z) \\
=E \Psi(x, y, z) &
\end{aligned}
$$

where $z$-dimension is chosen perpendicular to the tunnelling barriers, and $x$ - and $y$-dimensions parallel to them. In Eq. (1) $V(x, y, z)$ is the $3 \mathrm{D}$ potential distribution which, in general, consists of the lateral confinement potential, $V_{\text {confinement }}(x, y, z)$, the electron affinity, $V_{\text {affinity }}(z)$, along the vertical channel due to different layered materials, and the other elastic scattering potentials, $V_{\text {irregular }}(x, y, z)$, due to all irregularities like ionised impurities or interface roughness:

$V(x, y, z)=V_{\text {confinement }}(x, y, z)+V_{\text {affinity }}(z)+V_{\text {irregular }}(x, y, z)$.

The 3D wavefunction, $\Psi(x, y, z)$, is decomposed using a complete set of 2D lateral eigenstates at each $z$ point, $\varphi_{\gamma}(x, y \mid z)$, as follows:

$\Psi(x, y, z)=\sum_{\gamma} \varphi_{\gamma}(x, y \mid z) \chi_{\gamma}(z)$

$\varphi_{\gamma}(x, y \mid z)$ is obtained by solving the following 2D Schrödinger equation:

$$
\begin{aligned}
- & \frac{\hbar^{2}}{2 m^{*}}\left(\frac{\partial^{2}}{\partial x^{2}}+\frac{\partial^{2}}{\partial y^{2}}\right) \varphi_{\gamma}(x, y \mid z)+V_{\text {confinement }}(x, y, z) \varphi_{\gamma}(x, y \mid z) \\
= & \epsilon_{\gamma}(z) \varphi_{\gamma}(x, y \mid z)
\end{aligned}
$$

with the Dirichlet boundary conditions, $\varphi_{\gamma}=0$, on the lateral surface of the device. The index, $\gamma$, represents a 2D lateral mode number and $\epsilon_{\gamma}(z)$ the corresponding $\gamma$ th lateral eigen-energy. In the numerical calculations Eq. (4) is discretised by using a 3D finite-difference mesh to obtain $\boldsymbol{\epsilon}_{\gamma}(z)$ and $\varphi_{\gamma}(x, y \mid z)$ along the vertical channel. Eq. (4) is solved by using the bisection method following Householder's tridiagonalisation to obtain $\epsilon_{\gamma}(z)$. The corresponding eigenstates, $\varphi_{\gamma}(x, y \mid z)$, are then calculated by the inverse iteration method. To make finding the eigenstates faster, it is very effective to adopt the set of eigenstates obtained at the present $z$-mesh point as an initial guess for the eigenstates at the next $z$-mesh point. In practice, a cut-off value is introduced for the maximum eigenenergy although all of lateral modes would be necessary to make a complete set. The number of eigenstates required for realistic calculations depends on the system under consideration, but, in general, the maximum lateral eigenenergy is chosen to be much larger than the Fermi energy in the contact regions.

From Eqs. (1) and (3), the following 1D scattering equation with lateral-mode mixing terms is derived for

$$
\begin{aligned}
& \chi_{\gamma}(z): \\
& \frac{\mathrm{d}^{2}}{\mathrm{~d} z^{2}} \chi_{\gamma}(z)+k_{\gamma}^{2}(z) \chi_{\gamma}(z)+\sum_{\gamma^{\prime}}\left\{2 C_{\gamma, \gamma^{\prime}}^{(0,1)}(z) \frac{\mathrm{d}}{\mathrm{d} z} \chi_{\gamma^{\prime}}(z)\right. \\
& \left.\quad+C_{\gamma, \gamma^{\prime}}^{(0,2)}(z) \chi_{\gamma^{\prime}}(z)-\frac{2 m^{*}}{\hbar^{2}} V_{\gamma, \gamma^{\prime}}^{\text {irregular }}(z) \chi_{\gamma^{\prime}}(z)\right\}=0
\end{aligned}
$$

where $k_{\gamma}(z)$ denotes a complex wave number given by

$k_{\gamma}(z)=\frac{\sqrt{2 m^{*}\left(E-\epsilon_{\gamma}(z)-V_{\text {affinity }}(z)\right)}}{\hbar}$

Two z-dependent matrices, $C^{(0,1)}(z)$ and $C^{(0,2)}(z)$, in Eq. (5) represent lateral mode mixing due to non-uniform lateral confinement at $z$ and are given by

$C_{\gamma, \gamma^{\prime}}^{(0,1)}=\iint \varphi_{\gamma}(x, y \mid z) \frac{\partial \varphi_{\gamma^{\prime}}(x, y \mid z)}{\partial z} \mathrm{~d} x \mathrm{~d} y$

$C_{\gamma, \gamma^{\prime}}^{(0,2)}=\iint \varphi_{\gamma}(x, y \mid z) \frac{\partial^{2} \varphi_{\gamma^{\prime}}(x, y \mid z)}{\partial z^{2}} \mathrm{~d} x \mathrm{~d} y$

and also the irregularity-induced scattering matrix, $V^{\text {irregular }}(z)$, is defined as follows:

$V_{\gamma, \gamma^{\prime}}^{\text {irregular }}(z)=\iint \varphi_{\gamma}(x, y \mid z) V_{\text {irregular }}(x, y \mid z) \varphi_{\gamma^{\prime}}(x, y \mid z) \mathrm{d} x \mathrm{~d} y$

These mode-mixing matrix elements in Eqs. (7)-(9) are numerically evaluated using the obtained set of $\varphi_{\gamma}(x, y \mid z)$. The first derivative term of $\chi_{\gamma}(z)$ in Eq. (5) can be eliminated by applying the relevant unitary transformation:

$\chi_{\gamma}(z)=\sum_{\gamma^{\prime}} M_{\gamma, \gamma^{\prime}}(z) f_{\gamma^{\prime}}(z)$

where the unitary matrix, $M(z)$, is defined using $C^{(0,1)}$ as follows:

$\left.M_{\gamma, \gamma^{\prime}}(z)=\exp \left(-\int^{z} C_{\gamma, \gamma^{\prime}}^{(0,1)}\left(z^{\prime}\right) \mathrm{d} z^{\prime}\right)\right)$

The unitary matrix, $M(z)$, is calculated using a secondorder expansion approximation which guarantees the unitarity of the matrix

$M(z) \cong\left\{1-\frac{1}{2} \int^{z} C^{(0,1)}\left(z^{\prime}\right) \mathrm{d} z^{\prime}\right\} \cdot\left\{1+\frac{1}{2} \int^{z} C^{(0,1)}\left(z^{\prime}\right) \mathrm{d} z^{\prime}\right\}^{-1}$

Substituting Eqs. (10) and (11) into Eq. (5), the transformation leads to the following equation:

$\frac{\mathrm{d}^{2}}{\mathrm{~d} z^{2}} f_{\gamma}(z)=-\sum_{\gamma^{\prime}} \omega_{\gamma, \gamma^{\prime}}(z) f_{\gamma^{\prime}}(z)$

where the matrix, $\omega(z)$, is written as follows:

$\omega_{\gamma, \gamma^{\prime}}(z)=\sum_{\gamma^{\prime \prime}} \sum_{\gamma^{\prime \prime \prime}}\left(M^{-1}\right)_{\gamma, \gamma^{\prime \prime}}(z) W_{\gamma^{\prime \prime}, \gamma^{\prime \prime \prime}}(z) M_{\gamma^{\prime \prime \prime}, \gamma^{\prime}}(z)$ 
The matrix, $W(z)$, in Eq. (14) is given by the following expressions:

$$
\begin{aligned}
W_{\gamma, \gamma^{\prime}}(z)= & k_{\gamma}^{2}(z) \delta_{\gamma, \gamma^{\prime}}-\left\{C^{(0,1)}(Z)\right\}_{\gamma, \gamma^{\prime}}^{2}-C_{\gamma, \gamma^{\prime}}^{(1,1)}(z) \\
& -\frac{2 m^{*}}{\hbar^{2}} V_{\gamma, \gamma^{\prime}}^{\text {irregular }}(z) \\
C_{\gamma, \gamma^{\prime}}^{(1,1)}(z)= & \iint \frac{\partial \varphi_{\gamma}(x, y \mid z)}{\partial z} \frac{\partial \varphi_{\gamma^{\prime}}(x, y \mid z)}{\partial z} \mathrm{~d} x \mathrm{~d} y
\end{aligned}
$$

Renormalised complex wave numbers, $K_{\gamma}(z)$, are obtained by solving the following eigenvalue equation:

$$
\sum_{\gamma^{\prime \prime}} W_{\gamma, \gamma^{\prime \prime}}(z) U_{\gamma^{\prime \prime}, \gamma^{\prime}}(z)=\left\{K_{\gamma^{\prime}}(z)\right\}^{2} U_{\gamma, \gamma^{\prime}}(z)
$$

where $U(z)$ is a unitary matrix which diagonalises the matrix $W(z)$. The eigenvalue equation Eq. (17) is discretised and numerically solved using the QL method. Then $\chi_{\gamma}(z)$ can be expressed as a superposition of plane waves:

$$
\begin{aligned}
\chi_{\gamma}(z)= & \sum_{\gamma^{\prime}} \sum_{\gamma^{\prime \prime}} M_{\gamma, \gamma^{\prime}}(z) U_{\gamma^{\prime}, \gamma^{\prime \prime}}(z)\left[A_{\gamma^{\prime \prime}}(z) \exp \left\{i K_{\gamma^{\prime \prime}}(z) z\right\}\right. \\
& \left.+B_{\gamma^{\prime \prime}}(z) \exp \left\{-i K_{\gamma^{\prime \prime}}(z) z\right\}\right]
\end{aligned}
$$

where $A_{\gamma}(z)$ and $B_{\gamma}(z)$ are coefficients of forward and backward plane waves in the $\gamma$ th lateral mode with the complex wave number $K_{\gamma}(z)$. Assuming these coefficients to be constant between two adjacent $z$-mesh points, the 3D wavefunction, $\Psi(x, y, z)$, can be finally written as

$$
\begin{aligned}
\Psi^{(i)}(x, y, z) \cong & \sum_{\gamma} \sum_{\gamma^{\prime}} \sum_{\gamma^{\prime \prime}} \varphi_{\gamma}(x, y \mid z) M_{\gamma, \gamma^{\prime}}^{(i)} U_{\gamma^{\prime}, \gamma^{\prime \prime}}^{(i)}\left[A_{\gamma^{\prime \prime}}^{(i)} \exp \left\{i K_{\gamma^{\prime \prime}}^{(i)} z\right\}\right. \\
& \left.+B_{\gamma^{\prime \prime}}^{(i)} \exp \left\{-i K_{\gamma^{\prime}}^{(i)} z\right\}\right]
\end{aligned}
$$

where the index $(i)$ denotes a region between adjacent $z$ mesh points $z_{i}$ and $z_{i+1}$. From the continuity of electron probability flux of electrons through the device, the coefficients at the adjacent $z$-mesh points are then related as follows:

$$
\left(\begin{array}{c}
A_{\gamma}^{(i+1)} \\
B_{\gamma}^{(i+1)}
\end{array}\right)=\sum_{\gamma^{\prime}} T^{(i)}\left(\gamma, \gamma^{\prime}\right)\left(\begin{array}{c}
A_{\gamma^{\prime}}^{(i)} \\
B_{\gamma^{\prime}}^{(i)}
\end{array}\right)
$$

The multi-mode transfer matrix, $T^{(i)}$, can be expressed as $T^{(i)}\left(\gamma, \gamma^{\prime}\right)=\left(\begin{array}{cc}\alpha_{+}^{(i)}\left(\gamma, \gamma^{\prime}\right) \cdot P^{(i)} & \alpha_{-}^{(i)}\left(\gamma, \gamma^{\prime}\right) \cdot\left(Q^{(i)}\right)^{-1} \\ \alpha_{-}^{(i)}\left(\gamma, \gamma^{\prime}\right) \cdot Q^{(i)} & \alpha_{+}^{(i)}\left(\gamma, \gamma^{\prime}\right) \cdot\left(P^{(i)}\right)^{-1}\end{array}\right) \cdot X_{\gamma, \gamma^{\prime}}^{(i)}$

where the matrices, $\alpha^{(i)}$ and $X^{(i)}$, are given by the following equations:

$$
\begin{aligned}
& \alpha_{ \pm}^{(i)}\left(\gamma, \gamma^{\prime}\right)=\frac{1}{2}\left\{1 \pm \frac{m^{*(i+1)}}{m^{*(i)}} \frac{K_{\gamma^{\prime}}^{(i)}}{K_{\gamma}^{(i+1)}}\right\} \\
& X_{\gamma, \gamma^{\prime}}^{(i)}=\sum_{\gamma_{1}} \sum_{\gamma_{2}} \sum_{\gamma_{3}} U_{\gamma_{1}, \gamma}^{(i+1)} M_{\gamma_{2}, \gamma_{1}}^{(i+1)} M_{\gamma_{2}, \gamma_{3}}^{(i)} U_{\gamma_{3}, \gamma^{\prime}}^{(i)}
\end{aligned}
$$

and $P^{(i)}$ and $Q^{(i)}$ stand for

$$
P^{(i)}=\exp \left\{i\left(K_{\gamma^{\prime}}^{(i)}-K_{\gamma}^{(i+1)}\right) z_{i+1}\right\}
$$

$Q^{(i)}=\exp \left\{i\left(K_{\gamma^{\prime}}^{(i)}+K_{\gamma}^{(i+1)}\right) z_{i+1}\right\}$

Hence the coefficients at the emitter and collector edges of the device are related by using a multi-mode transfer matrix, $T\left(\gamma, \gamma^{\prime}\right)$ :

$$
\begin{aligned}
& T=T^{(N)} \cdot T^{(N-1)} \cdot T^{(N-2)} \cdots T^{(2)} \cdot T^{(1)} \\
& \left(\begin{array}{c}
A_{\gamma}^{\text {Emitter }} \\
B_{\gamma}^{\text {Emitter }}
\end{array}\right)=\sum_{\gamma^{\prime}} T\left(\gamma, \gamma^{\prime}\right)\left(\begin{array}{l}
A_{\gamma^{\prime}}^{\text {Collector }} \\
B_{\gamma^{\prime}}^{\text {Collector }}
\end{array}\right)
\end{aligned}
$$

It should be noted that the transfer matrix contains both propagating and evanescent modes depending on the total energy and lateral mode eigenenergies. In the following calculations the full transfer matrix is adopted including the evanescent modes in order to make the transfer matrix regular even under a non-zero external bias. A relevant multi-mode scattering matrix, $S\left(\gamma, \gamma^{\prime}\right)$, which is defined as

$$
\left(\begin{array}{c}
B_{\gamma}^{\text {Emitter }} \\
A_{\gamma}^{\text {Collector }}
\end{array}\right)=\sum_{\gamma^{\prime}} S\left(\gamma, \gamma^{\prime}\right)\left(\begin{array}{c}
A_{\gamma^{\prime}}^{\text {Emitter }} \\
B_{\gamma^{\prime}}^{\text {Collector }}
\end{array}\right)
$$

is calculated from the transfer matrix. The total transmission rate is then obtained from the S-matrix as follows:

$$
\begin{gathered}
T(E)=\sum_{\gamma} \sum_{\gamma^{\prime}} t_{\text {Collector }}\left(\gamma, \gamma^{\prime} ; E\right) \theta\left(E-\epsilon_{\gamma}^{\text {Emitter }}\right) \theta\left(E-\epsilon_{\gamma^{\prime}}^{\text {Collector }}\right) \\
=\sum_{\gamma} \sum_{\gamma^{\prime}} t_{\text {Emitter }}\left(\gamma, \gamma^{\prime} ; E\right) \theta\left(E-\epsilon_{\gamma}^{\text {Emitter }}\right) \theta\left(E-\epsilon_{\gamma^{\prime}}^{\text {Collector }}\right)
\end{gathered}
$$

where $\theta(E)$ is the step function, and the multi-mode transmission probabilities are defined as follows:

$t_{\text {Collector }}\left(\gamma, \gamma^{\prime} ; E\right)=\left|S_{12}\left(\gamma, \gamma^{\prime}\right)\right|^{2}$

$t_{\text {Emitter }}\left(\gamma, \gamma^{\prime} ; E\right)=\left|S_{21}\left(\gamma, \gamma^{\prime}\right)\right|^{2}$

A complete set of the 3D wavefunctions, $\Psi(x, y, z)$, can be obtained by using the following scattering boundary conditions:

$\left(A_{\gamma}^{\text {Emitter }}, B_{\gamma}^{\text {Collector }}\right)=\left(0, \delta_{\gamma, \gamma_{0}}\right)$

for an incident electron-wave with a lateral mode index, $\gamma_{0}$, 
(a)

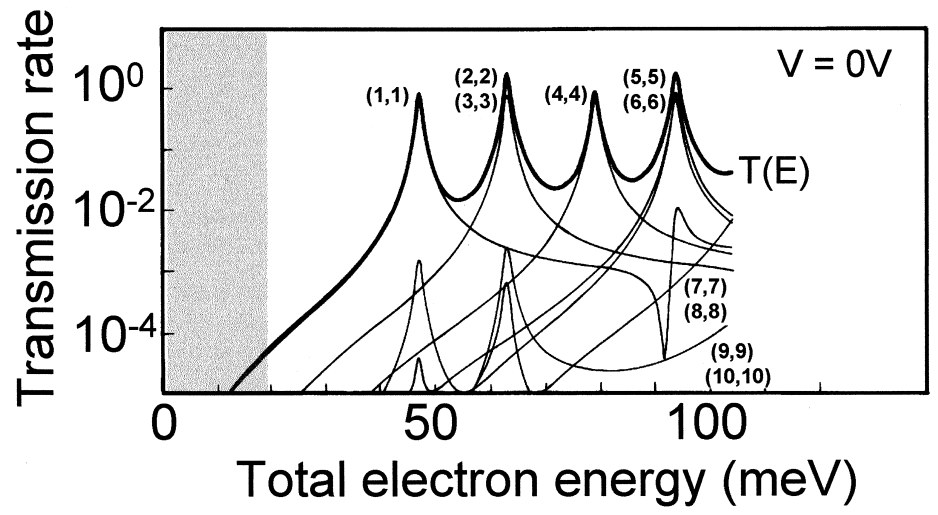

(b)

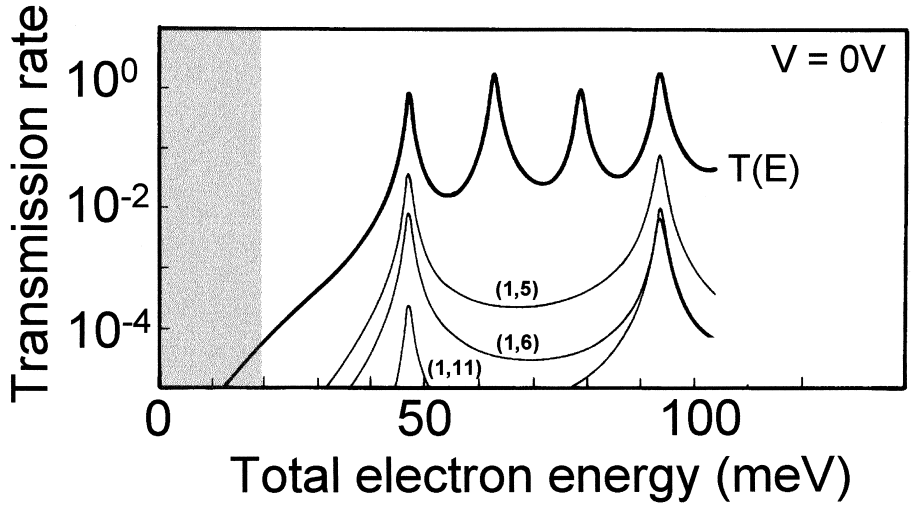

Fig. 3. Multi-mode transmission rates calculated for a 0D RTD structure shown in Fig. 1(a): (a) total electron energy dependence of the transmission rates for lateral-mode conserving tunnelling, $(\gamma, \gamma)$; and (b) that for lateral-mode non-conserving tunnelling, $\left(1, \gamma^{\prime}\right)$. The emitter pad size of the 0D RTD is assumed to be $80 \times 80 \mathrm{~nm}^{2}$.

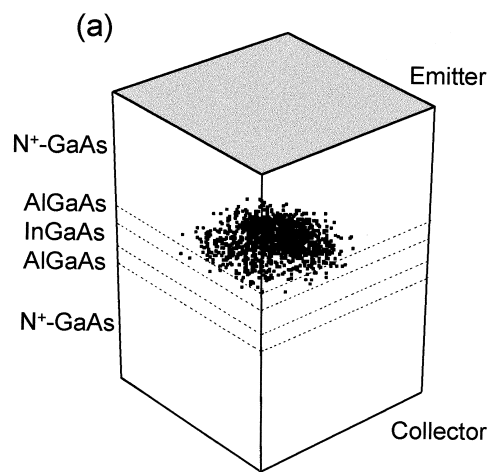

(b)

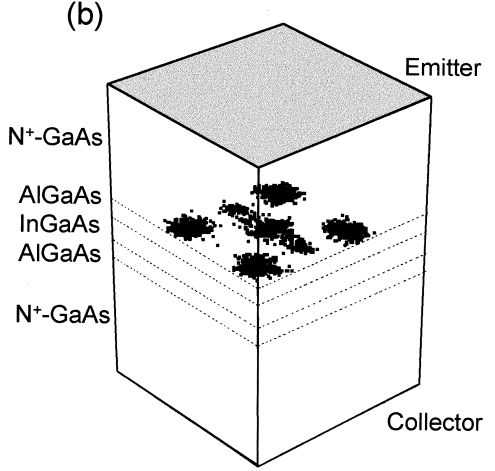

Fig. 4. Visualised 3D probability density of electrons simulated for the incident mode at (a) the first resonance and (b) the fourth resonance energies in Fig. 3. originating at the emitter edge of the device, and

$\left(A_{\gamma}^{\text {Emitter }}, B_{\gamma}^{\text {Collector }}\right)=\left(\delta_{\gamma, \gamma_{0}}, 0\right)$

when it originates at the collector edge. In Eqs. (32) and (33) $\delta_{\gamma, \gamma_{0}}$ is the delta function. Finally, total tunnelling current, $I_{\text {tunnel }}$, is calculated assuming the global coherent tunnelling of electrons [13] throughout the device as follows:

$$
\begin{aligned}
I_{\text {tunnel }}= & \frac{e}{\pi \hbar} \int_{E_{c}^{\text {Emitter }}}^{\infty} T(E)\left\{f_{\text {Emitter }}\left(E ; E_{F}^{\text {Emitter }}\right)\right. \\
& \left.-f_{\text {Collector }}\left(E ; E_{F}^{\text {Collector }}\right)\right\} \mathrm{d} E
\end{aligned}
$$

where $f_{\text {Emitter }}$ and $f_{\text {Collector }}$ are Fermi distribution functions in the emitter and collector contact regions, respectively.

\section{Multi-mode resonant tunnelling and quasi-bound states in quantum dots}

In this section the simulation technique described in Section 3 is applied for analysing the effects of lateral confinement and mode-mixing on the $I-V$ characteristics. For this study the simple 2-terminal 0D RTD structure (Fig. 1(a)) with an $\mathrm{Al}_{0.25} \mathrm{Ga}_{0.75} \mathrm{As}(4 \mathrm{~nm}) / \mathrm{In}_{0.08} \mathrm{Ga}_{0.92} \mathrm{As}(5 \mathrm{~nm}) /$ $\mathrm{Al}_{0.25} \mathrm{Ga}_{0.75} \mathrm{As}(4 \mathrm{~nm}) \mathrm{DB}$ structure is used. The DB structure is sandwiched with two undoped GaAs spacer layers of $6 \mathrm{~nm}$ in thickness, and $\mathrm{n}^{+}$-type GaAs emitter and collector 

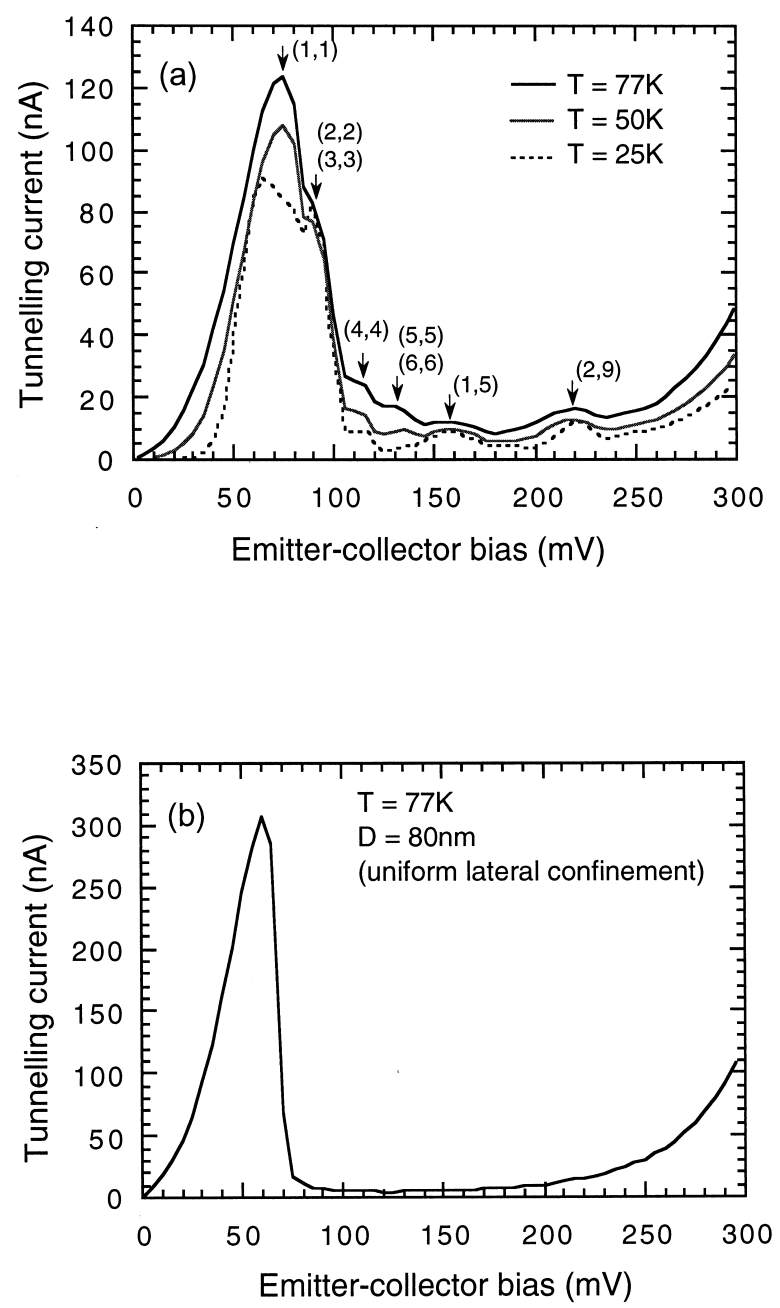

Fig. 5. (a) Emitter-collector voltage dependence of total tunnelling current calculated at various temperatures for the 0D RTDs with hour-glass-shaped confinement potential. Current peaks and shoulders are indicated by arrows with the indices, $\left(\gamma, \gamma^{\prime}\right)$, for the corresponding main tunnelling processes. (b) $I-V$ characteristics calculated by assuming a completely uniform lateral confinement for comparison.

layers with donor concentration of $1.0 \times 10^{18} \mathrm{~cm}^{-3}$. Lateral dimensions of the device are set to be $80 \mathrm{~nm}$ in both $x$ and $y$ dimensions. The quantum dot region is formed by using undoped InGaAs and so is assumed to be completely clean, i.e. $V_{\text {irregular }}(x, y, z)=0$. As explained in Section 2, an hour-glass-shaped non-uniform lateral confinement potential, $V_{\text {confinement }}(x, y, z)$, is formed in this structure, and, strictly speaking, it should be determined through a self-consistent calculation. However, this would require an enormous amount of computational time, and so, in this work, $V_{\text {confinement }}(x, y, z)$ is calculated by using a classical drift-diffusion-type device simulation [24] in which surface carrier traps are taken into consideration by using Spicer's unified defect model [25]. As long as the size of the lateral confinement is much larger than the width of the quantum well, the calculated potential distribution should be a fairly good approximation.

The multi-mode transmission probability calculated for the OD RTD structure is shown in Fig. 3. Fig. 3(a) and (b) show the total energy dependence of transmission rates at zero emitter-collector voltage. In Fig. 3(a) the lateral-mode conserving tunnelling, $\left|S_{12}(\gamma, \gamma)\right|^{2}$, in which the lateralmode number $\gamma$ is conserved during tunnelling is shown by using thin solid lines. The total transmission rate $T(E)$ is also shown by a thick solid line. A grey area in the figures is the Fermi sea in the emitter contact, and the indices $(\gamma$, $\left.\gamma^{\prime}\right)$ represent the tunnelling process from the $\gamma$ th mode in the emitter to the $\gamma^{\prime}$ th mode in the collector. As shown in Fig. 3(a) multiple transmission peaks in $T(E)$ are mainly composed of the transmission peaks of the lateral-mode conserving tunnelling. The 3D existent probability of electrons, $\left|\Psi_{E}(x, y, z)\right|^{2}$, calculated for the first incident mode $(\gamma=1)$ at the first resonant energy is plotted in Fig. 4(a), which shows the nature of the lowest quasi-bound state of the quantum dot.

Owing to the non-uniform confinement potential, however, the S-matrix has non-zero off-diagonal elements, which represent the tunnelling processes with a change in the lateral-mode. Fig. 3(b) shows the total energy dependence of transmission rates for such a lateral-mode nonconserving tunnelling, $\left|S_{12}\left(1, \gamma^{\prime}\right)\right|^{2}$, for the first incident mode. In Fig. 3(b) the tunnelling with the first incident mode is observed only for the outgoing mode $\gamma^{\prime}=5,6$, and 11 . This is purely because of a selection rule for parity of lateral wavefunctions. Because the elastic scattering due to the hour-glass confinement potential does not break symmetry under mirror reflection in $x$ and $y$ dimensions, a lateral mode couples only with other modes having the same parity. The lowest wavefunction has even parities in both $x$ and $y$ dimensions, and can therefore couple only with upper modes described above. It can be seen that the transmission rates for the lateral-mode non-conserving tunnelling also show resonance peaks at 47 and $93 \mathrm{meV}$. These enhanced transmission probability in the off-diagonal processes lead to additional structures in the diagonal processes in Fig. 3(a): a Fano-resonance-type asymmetric resonance [26] can be seen for $(1,1)$ at $93 \mathrm{meV}$. This structure stems from the enhanced probability of the second order diagonal tunnelling, $1 \rightarrow 5 \rightarrow 1$, The 3D existent probability of electrons, $\left|\Psi_{E}(x, y, z)\right|^{2}$, calculated for the first incident mode at $92 \mathrm{meV}$ (at the dip of the rate) is shown in Fig. 4(b). It should be noted that the electron existent probability in the quantum dot reflects features of the fifth-mode rather than first-mode despite the incoming wave with the first mode. This signifies that a large part of the incoming wave is converted to the fifth-mode in the quantum dot. In other words, the lateral mode index $\gamma$ is no longer a good quantum number for this system.

Fig. 5(a) shows the emitter-collector voltage dependence of the total tunnelling current at a temperature of 25,50 and $77 \mathrm{~K}$. Several satellite current peaks and shoulders are observed superposed on the conventional NDC characteristics. For comparison, the current-voltage characteristic calculated assuming the uniform confinement is also 
(a)

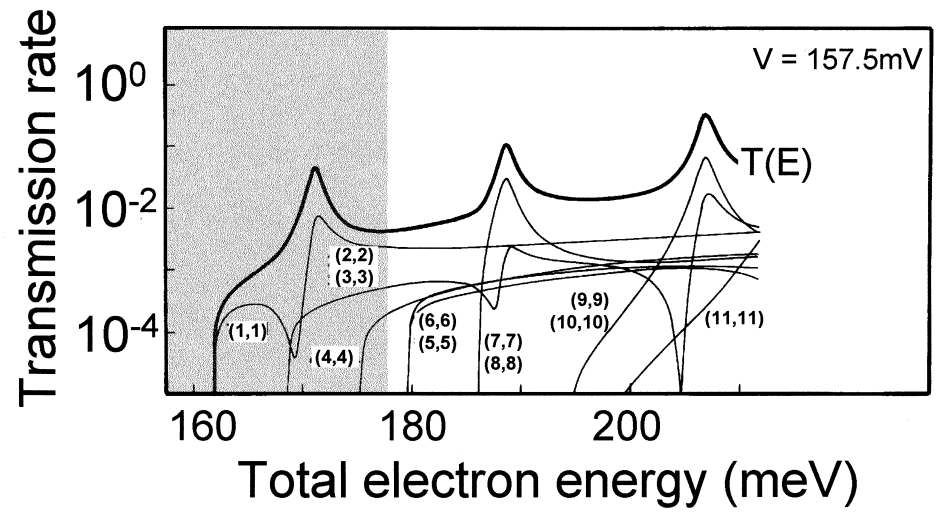

(b)

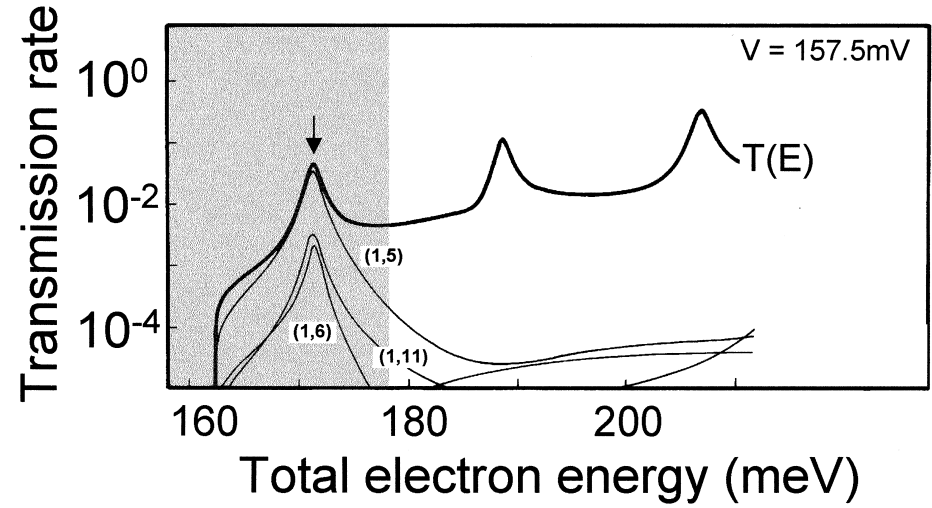

Fig. 6. Total electron energy dependence of the transmission rates calculated at the fifth peak voltage, $V=157.5 \mathrm{mV}$, in Fig. 3(a): (a) for lateral-mode conserving tunnelling, $(\gamma, \gamma)$; and (b) that for lateral-mode non-conserving tunnelling, $\left(1, \gamma^{\prime}\right)$.

shown in Fig. 5(b). It should be noted that only one main current peak is found without any fine structure in the case of the uniform confinement. As indicated in Fig. 5(a), first four peaks in the $I-V$ curves mainly result from the lateralmode conserving resonant tunnelling: $(1,1)$ tunnelling for the first main peak, $(2,2)$ and $(3,3)$ for the second, $(4,4)$ for the third, and $(5,5)$ and $(6,6)$ for the fourth. As shown in Fig. 5(a) the fourth peak becomes smaller when temperature is decreased as it is caused by the tunnelling of electrons which are thermally excited to the fifth and sixth modes located above the quasi-Fermi level in the emitter region.

However, the last two current peaks in Fig. 5(a) were found to be caused by the lateral-mode non-conserving tunnelling. The total energy dependence of the transmission probability calculated at the fifth peak voltage $(V=$ $157.5 \mathrm{mV}$ ) is shown in Fig. 6(a) and (b): (a) is the diagonal $\left|S_{12}(\gamma, \gamma)\right|^{2}$; and (b) the off-diagonal $\left|S_{12}(1, \gamma)\right|^{2}$. At this peak voltage a small transmission peak located under the Fermi energy leads to the fifth current peak, which is caused by the lateral-mode non-conserving tunnelling process $(1,5)$ (see Fig. 6(b)). From the same analysis it is found that the sixth current peak is also due to the lateral-mode non-conserving tunnelling process $(2,9)$.

As shown in this section, the present 3D S-matrix-based numerical simulation facilitates to investigate the complex lateral mode mixing in the quantum dot and the fine structures in the $I-V$ characteristics.

\section{Resonant tunnelling through localised states formed by random ionised impurities}

In Section 4 the 0D RTD with a completely clean quantum dot has been investigated. As the quantum dot area is generally formed with undoped semiconductors, the typical background doping concentration is of the order of $10^{14} \mathrm{~cm}^{-3}$. As far as the radius of the active quantum dot is of the order of a few tens nanometers and the quantum well width is of the order of a few nanometers, the probability of a single impurity being in the quantum dot will be very small. This section deals with quantum dots larger than those in the previous section, in which a single or a few background impurity atoms are contained.

For this analysis, the following simple model is used for $V_{\text {irregular }}(x, y, z)$ in Eq. (2) to introduce the random ionised impurity potential:

$V_{\text {irregular }}(x, y, z)=V_{\text {impurity }}(\boldsymbol{r})=\sum_{\boldsymbol{r}_{i}} V_{\text {im }}\left(\boldsymbol{r}-\boldsymbol{r}_{i}\right)$

where $V_{\mathrm{im}}$ is expressed by the delta functional potential due to a discrete ionised impurity, and $\left\{\boldsymbol{r}_{i}\right\}$ denotes the spatial configuration of the ionised impurities. The 3D spatial coordinates, $\boldsymbol{r}_{i}$, of ionised donors are expressed using lateral and vertical displacement from the centre of the quantum $\operatorname{dot},(\delta x, \delta y, \delta z)$.

The numerical simulation was performed for a relatively 


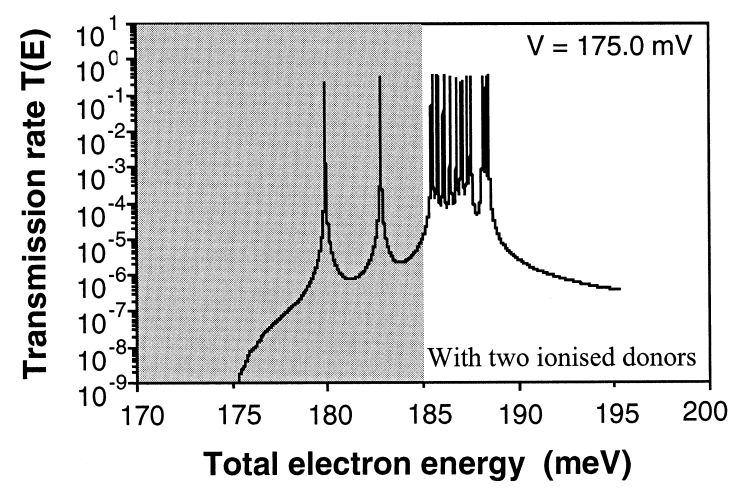

Fig. 7. Total electron energy dependence of the total transmission rate calculated for a large-area (200 nm in diameter) OD RTD with two ionised donors placed in the quantum dot. The emitter-collector voltage is $175 \mathrm{mV}$ which corresponds to the second plateau of the $I-V$ characteristics (see Fig. 9).

large-area 0D RTD with the quantum dot region composed of a symmetric $\operatorname{AlAs}(4.2 \mathrm{~nm}) / \mathrm{GaAs}(5.6 \mathrm{~nm}) / \operatorname{AlAs}(4.2 \mathrm{~nm})$ DB structure. The corresponding experimental results were obtained for the structure shown in Fig. 1(b), which achieves a virtually flat lateral confinement around the DB structure $[13,19]$. In this section, therefore, only the essential active device region is considered, and the lateral confinement is assumed to be completely flat. The confinement profile is simply modelled using a square well $200 \mathrm{~nm}$ in diameter in which the intervals of lateral eigenenergies are so small that contributions from different modes are barely separable on the $I-V$ curve.

We examine the device which contains two ionised donors: one donor is at the centre of the active device region, $(\delta x, \delta y, \delta z)=(0,0,0)$ and the other at $(\delta x, \delta y, \delta z)=$ (48, 48 and $1.3 \mathrm{~nm}$, respectively). Fig. 7 shows the energy dependence of the total transmission rate, $T(E)$, for an emitter-collector voltage of $175 \mathrm{mV}$. In the high-energy region we see a series of transmission peaks which are very close to each other. These transmission peaks represent RT through

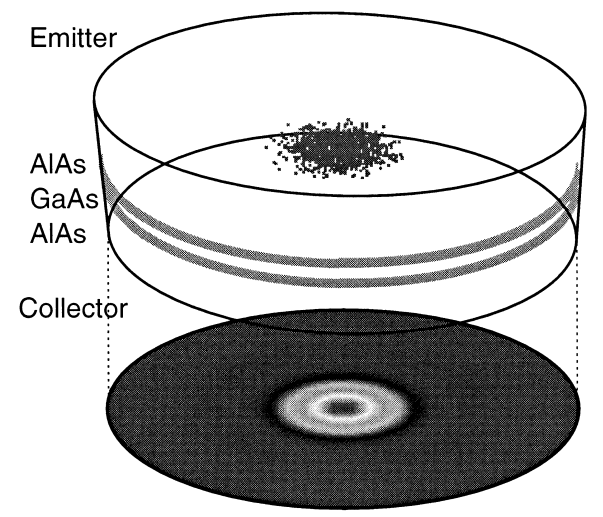

(a) the original quantised states whose energy intervals are determined by the size of the lateral confinement and which are thus nearly degenerate as the confinement size is chosen to be fairly large. These peaks simply give rise to a large single NDC in the $I-V$ characteristics (see the main peak in the inset of Fig. 9) in the same way as in conventional RTDs. In addition to these peaks, however, two separate transmission peaks are seen in Fig. 7 at lower energies. These new peaks result from the ionised donors embedded in the active device region: the attractive potential of each ionised donor selectively pulls down one of the degenerate states to lower energy. The state chosen is determined by both the position of the ionised donor and the spatial distribution of the original eigenstate. This may be understood from the probability densities of electrons calculated at these two resonant energies. Fig. 8(a) and (b) show the visualised $3 \mathrm{D}$ and $2 \mathrm{D}$ probability densities at (a) first and (b) second resonances calculated for the lowest first-mode incident waves from the emitter. The $2 \mathrm{D}$ pictures show the in-plane amplitude at $z=0,|\Psi(x, y, 0)|^{2}$. It can be seen that the electron probability densities are strongly localised around the different ionised donor sites although the incident wave spreads laterally over the whole area at the emitter edge.

These transmission characteristics lead to the $I-V$ characteristics shown in Fig. 9. It is found that the ionised-donor-induced transmission peaks seen in Fig. 7 lead to current plateaux with a step, $\Delta I$, of about $0.1 \mathrm{nA}$ before the main NDC. Obviously the details of the current plateaux depend on the donor configuration, and the $I-V$ curve shown here is just for one particular configuration. It should, however, be noted that the simulated current step corresponds well to the experimental results $[13,19]$. This is because $\Delta I$ is essentially determined by the width of the corresponding transmission peak, i.e. the dwell time, $\tau_{\mathrm{d}}$, [13] of electrons at the resonant state, as follows:

$$
\Delta I=e / \tau_{\mathrm{d}}
$$

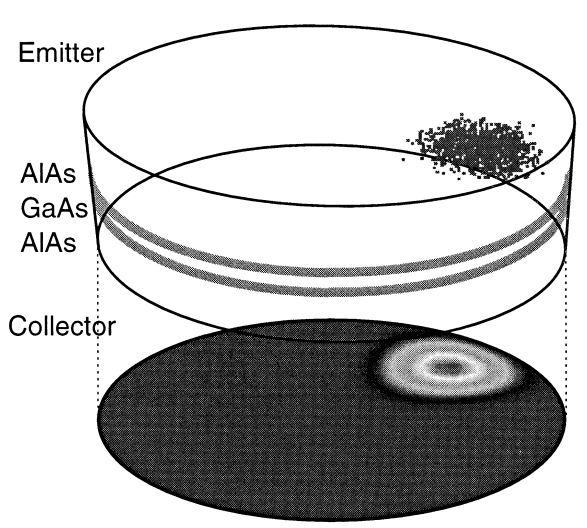

(b)

Fig. 8. Visualised 3D and 2D probability density of electrons simulated for the first incident mode at (a) the first resonance and (b) the second resonance energies in Fig. 7. 


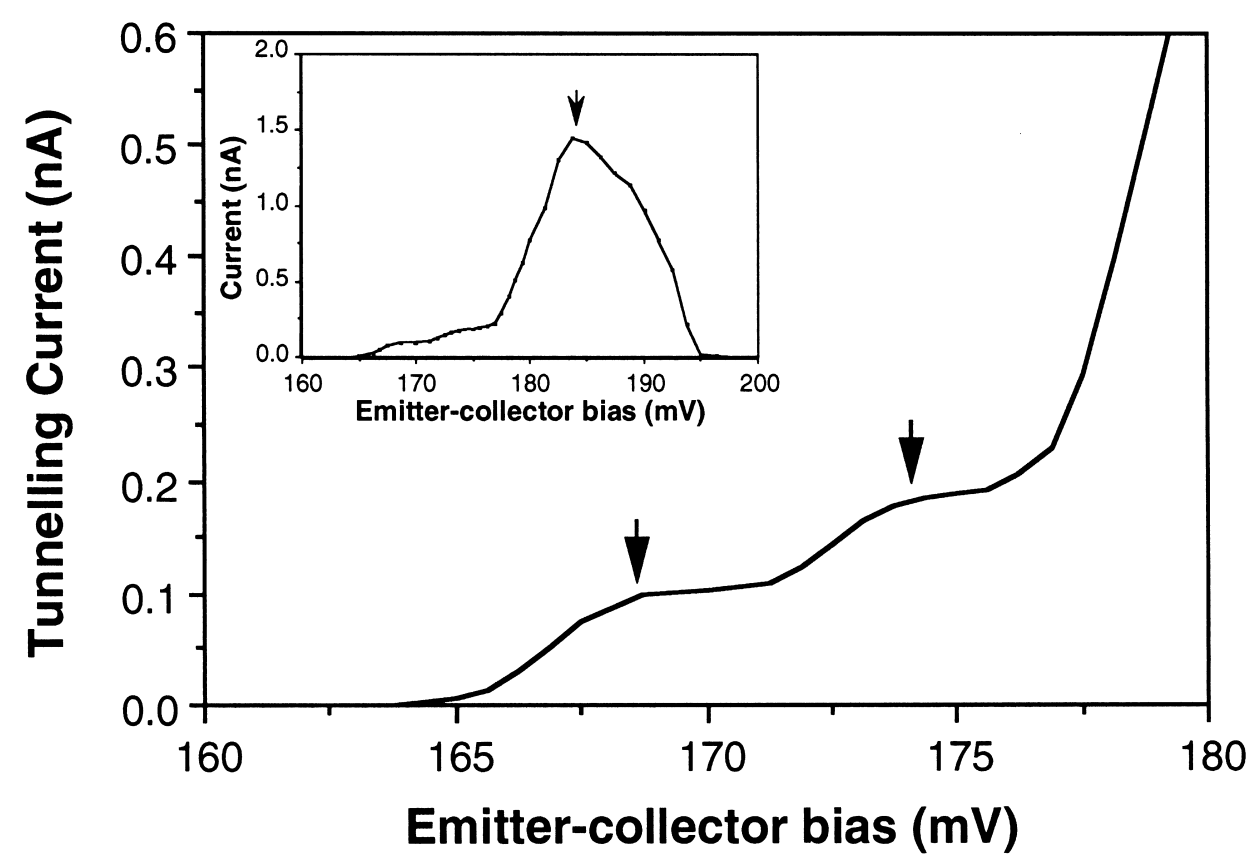

Fig. 9. Current plateaux in the $I-V$ curve calculated at $4.2 \mathrm{~K}$ for a large-area $0 \mathrm{D}$ RTD with two ionised donors in the quantum dot. The inset shows the full $I-V$ characteristics of the device.

where $\tau_{\mathrm{d}}$ is mainly determined by the layer thicknesses of the AlAs/GaAs/AlAs DBs. The dwell time estimated from Eq. (36) is also found to be in good agreement with that evaluated for the present OD RTD by magneto-conductance measurements [13,27].

The detailed fine structure of the $I-V$ characteristics depends on the location of the ionised donor in the quantum dot. From the analysis by using a single donor ion [15], it was found that the single transmission peak induced by the single ionised donor gradually shifts to higher energy and finally merges into the series of peaks when the donor is moved from the centre to the edge of the quantum dot, either laterally or vertically. This is because the interactions between the single ionised donor and the tunnelling electrons are essentially determined by the overlap of the original lateral wavefunctions with the local potential profiles. As the single donor moves towards the edge the amplitude of the original lateral wavefunction at the donor site decreases, and the attractive potential of the donor has less effect on the electron state. As a result the subthreshold voltage of the single current plateau also shifts towards the main peak voltage with a positional shift of the single donor while $\Delta I$ remains virtually the same. In the case of multiple ionised donors, therefore, the fine structure will be given by a superposition of the current plateaux and so will be donorconfiguration-dependent.

\section{Conclusion}

We have presented the numerical simulation based on the 3D multi-mode S-matrix theory as a powerful analysis tool for quantum-dot-based electronic devices. The simulation technique has been applied for various vertical OD RTDs. The effects of the lateral-mode non-conserving RT processes on the $I-V$ characteristics were investigated. The calculated S-matrix clearly showed new transmission peaks in the off-diagonal components, which result in the extra satellite current peaks. The simulation has also been applied for analysing RT assisted by background ionised impurities in a quantum dot. It has been demonstrated that a single ionised donor impurity forms a new quasi-bound state at a lower energy than other levels and leads to a current step before the main current threshold, which is in good agreement with the experimental data.

\section{Acknowledgements}

The author wishes to thank Drs M. Wagner and K. Nakazato of the Hitachi Cambridge Laboratory, Hitachi Europe Ltd., and Dr T. Teshima of Central Research Laboratory, Hitachi Ltd., for invaluable discussions.

\section{References}

[1] M.A. Reed, J.N. Randall, R.J. Aggarwal, R.J. Matyi, T.M. Moore, A.E. Westel, Observation of discrete electronic states in a zerodimensional semiconductor nanostructure, Phys. Rev. Lett. 60 (6) (1988) 535.

[2] M.A. Reed, J.N. Randall, J.H. Luscombe, Non-equilibrium quantum dots: transport, Nanotechnology 1 (1990) 63.

[3] S. Tarucha, Y. Hirayama, T. Saku, T. Kimura, Resonant tunneling through one- and zero-dimensional states constricted by $\mathrm{Al}_{x} \mathrm{Ga}_{1-x} \mathrm{As} /$ $\mathrm{GaAs} / \mathrm{Al}_{x} \mathrm{Ga}_{1-x} \mathrm{As}$ heterojunctions and high-resistance regions 
induced by focused Ga ion-beam implantation, Phys. Rev. B41 (1990) 5459.

[4] B. Su, V.J. Goldman, M. Santos, Shayegan, Resonant tunneling in submicron double-barrier heterostructures, Appl. Phys. Lett. 58 (1991) 747.

[5] M.W. Dellow, P.H. Benton, M. Henini, P.C. Main, L. Eaves, S.P. Beaumont, C.D.W. Wilkinson, Gated resonant tunnelling devices, Electronics Lett. 27 (1991) 134.

[6] P. Gueret, N. Blanc, R. German, H. Rothuizen, Confinement and single-electron tunneling in Schottky-gated, laterally squeezed double-barrier quantum-well heterostructure, Phys, Rev. Lett. 68 (1992) 1896.

[7] S. Tarucha, T. Tokura, Y. Hirayama, Resonant tunneling of threedimensional electrons into degenerate zero-dimensional levels, Phys. Rev. B B44 (1991) 13815.

[8] D.G. Austing, T. Honda, S. Tarucha, New design for submicron double-barrier resonant tunnelling transistors, Semicond. Sci. Technol. 11 (1996) 212.

[9] S. Tarucha, D.G. Austing, T. Honda, R.J. van der Hage, L.P. Kouwenhoven, Shell filling and spin effects in a few electron quantum dot, Phys. Rev. Lett. 77 (1996) 3613.

[10] M.J. McLennan, Y. Lee, S. Datta, Voltage drop in mesoscopic systems: a numerical study using a quantum kinetic equation, Phys. Rev. B 43 (1991) 13 846. Also see S. Datta: Electronic Transport in Mesoscopic Systems, Cambridge University Press, Cambridge, 1995.

[11] H. Mizuta, C.J. Goodings, Quantum transport simulation based on the statistical density matrix, J. Phys.: Condens. Matter 3 (1991) 3739.

[12] F.A. Buot, K.L. Jensen, Lattice Weyl-Wigner formulation of exact many-body quantum-transport theory and applications to novel solidstate quantum-based devices, Phys. Rev. B 42 (1990) 9429.

[13] H. Mizuta, T. Tomonori, The Physics and Application of Resonant Tunnelling Diodes, Cambridge University Press, Cambridge, 1995.

[14] H. Mizuta, C.J. Goodings, M. Wagner, S. Ho, Three-dimensional numerical analysis of multi-mode quantum transport in zero-dimensional resonant tunnelling diodes, J. Phys.: Condens. Matter 4 (1992) 8783.
[15] H. Mizuta, Numerical study of single-electron resonant tunnelling via a few ionised donors in laterally confined resonant tunnelling diodes, Jpn. J. Appl. Phys. 35 (1996) 2012.

[16] M. Eto, Numerical studies of transport properties through artificial atoms and molecules, Solid-State Electron. 42 (1998) 1373.

[17] M. Eto, Anomalous temperature dependence of Coulomb oscillations at nearly degenerate levels, J. Phys. Soc. Jpn. 66 (1997) 2244.

[18] C.J. Goodings, H. Mizuta, J.R.A. Cleaver, H. Ahmed, Electron confinement in variable-area resonant tunnelling diodes using inplane implanted gates, Surf. Sci. 305 (1994) 353.

[19] C.J. Goodings, H. Mizuta, J.R.A. Cleaver, H. Ahmed, Variable-area resonant tunneling diodes using implanted in-plane gates, J. Appl. Phys. 76 (1994) 1276.

[20] R.L. Greene, K.K. Bajaj, Effect of magnetic fields on the energy levels of a hydrogenic impurity center in $\mathrm{GaAs} / \mathrm{Ga}_{1-x} \mathrm{Al}{ }_{x} \mathrm{As}$ quantum-well structures, Phys. Rev. B 34 (1986) 951.

[21] M. Luban, J.H. Luscombe, M.A. Reed, D.L. Pursey, Anharmonic oscillator model of a quantum dot nanostructure, Appl. Phys. Lett. 54 (1989) 1997.

[22] M. Tewordt, L. Martin-Moreno, J.T. Nicholls, M. Pepper, M.J. Kelly, V.J. Law, D.A. Ritchie, J.E.F. Frost, G.A.C. Jones, Phys. Rev. B 45 (1992) 14407.

[23] B. Su, V.J. Goldman, J.E. Cunningham, Single-electron tunneling in nanometer-scale double-barrier heterostructure devices, Phys. Rev. B 46 (1992) 7644.

[24] H. Mizuta, K. Yamaguchi, M. Yamane, T. Tanoue, S. Takahashi, Two-dimensional numerical simulation of Fermi-level pinning phenomena due to DX centers in AlGaAs/GaAs HEMTs, IEEE Trans. Electron. Device ED-36 (1989) 2307.

[25] W.E. Spicer, P.W. Chye, P.R. Skeath, C.Y. Su, I. Lindau, New and unified model for Schottky barrier and III-V insulator interface states formation, J. Vac. Sci. Technol. 16 (1979) 1422.

[26] U. Fano, Effects of configuration interaction on intensities and phase shifts, Phys. Rev. 124 (1961) 1866.

[27] C.J. Goodings, H. Mizuta, J.R.A. Cleaver, Electrical studies of charge build-up and phonon-assisted tunneling in double-barrier materials with very thick spacer layers, J. Appl. Phys. 75 (1994) 2291. 